\title{
Stefan-Type Free Boundary Problems for Heat Equations
}

\author{
By
}

\author{
Hideo KAWARADA*
}

\section{§1. Introduction}

The present paper is concerned with the Stefan problem for the heat equations, which arises, for instance, in the study of melting of ice adjacent to the heated water.

Free boundary problems for the heat equations have been considered for a century. Although in some special cases explicit solutions had happened to be known very early, existence theorems of general nature were proved first only twenty years ago in connection with the 1-dimensional Stefan problem by Rubinstein [1] and Dacev [2].

Since then, various papers on the Stefan problem have been published by many authors including Friedman [3], [10], Evans [4], J. Douglas \& Gallie [5], Sestini [6], Miranker [7], J. Douglas [8], Kyner [9], I. I. Kolodner [11], Ladyženskaya [12], Oleĭnic [13], Brezis [14], Nogi [15] and others.

Among their contributions we refer to the existence theorems due to Kyner, Friedman and Oleinnic which state the existence of solutions of the problem subject to the Dirichlet or Neumann boundary conditions imposed on the boundary of the heated water, under the assumption that even at the initial moment there does exist some water. As a matter of fact, the absence of water at the initial moment invoke a certain singularity or difficulty of the problem from the mathematical view point, which is rather mild for the case of the Neumann boundary condition but is quite hard to handle for the case of the Dirichlet boundary condition.

Indeed, Kyner [9] and Friedman [10] have succeeded in dealing with

Communicated by S. Matsuura, February 25, 1973. Revised August 8, 1973.

* Department of Applied Physics, University of Tokyo, Tokyo. 
the former case where the Neumann boundary condition is imposed and there is no water at the initial moment by means of contraction mapping and by means of a limiting procedure (also, see Problems $1 \sim 7$ of chap. 8 of Friedman [16]), respectively.

The objective of the present paper is to settle the remaining open case, that is, to prove an existence theorem for the Stefan problem in the case where the Dirichlet boundary condition is imposed and there is no water at the initial moment. The actual proof will be carried out by means of a limiting procedure apparently similar to that of Friedman [10] and by means of some crucial estimates of approximate solutions.

\section{§2. Notations and Results}

2.1. Before describing our results we have to introduce some concepts and notations. It seems to be convenient to begin with a brief reproduction of Friedman's formulation. His problem is to seek two functions $u=u(x, t)$ and $s=s(t)$ which satisfy the following equations:

$$
\begin{array}{ll}
u_{t}=u_{x x}, & 0<x<s(t), \quad t>0, \\
u(0, t)=f(t) \geqq 0, & t>0, \\
u(x, 0)=\psi(x) \geqq 0, & 0<x<b, \quad \psi(b)=0, \quad b>0, \\
u(s(t), t)=0, & t>0, \\
\frac{d s(t)}{d t}=-u_{x}(s(t), t), & t>0, \\
s(0)=b . &
\end{array}
$$

Here $u$ stands for the temperature of water and the equation $x=s(t)$ represents the free boundary. The conditions (2.2) (2.3) and (2.4) are the usually given data for the temperature whereas the additional condition (2.5a) (the equation of heat balance) is a condition on the free boundary $x=s(t)$. The assumptions $f \geqq 0$ and $\psi \geqq 0$ correspond to the fact that the temperature of water is non-negative. Hereafter this problem will be denoted by FBP. I. 
Definition 2.1. We say that $u, s$ form a solution of FBP. I for $0<t<\sigma(0<\sigma<+\infty)$ if the following conditions are satisfied:

(i) $u_{x x}$ and $u_{t}$ are continuous for $0<x<s(t), 0<t<\sigma$,

(ii) $u$ and $u_{x}$ are continuous for $0 \leqq x \leqq s(t), 0<t<\sigma$,

(iii) $u$ is continuous, also for $t=0,0<x \leqq b$ and $0 \leqq \lim \inf u(x, t) \leqq \lim \sup u(x, t)<+\infty$ as $t \rightarrow 0, x \rightarrow 0$,

(iv) $s(t)$ is continuously differentiable for $0 \leqq t<\sigma$,

(v) $u$ and $s$ satisfy FBP. I.

By Friedman the following theorem for FBP. I was given:

Theorem 2.2. In FBP. I, suppose that $f(t)(0 \leqq t<+\infty)$ and $\psi(x)$ $(0 \leqq x \leqq b)$ are continuously differentiable functions. Then there exists one and only one solution $u$ and $s$ of FBP. I for $0<t<+\infty$. Furthermore, the free boundary $s(t)$ is monotone nondecreasing in $t$.

We shall give an outline of the proof of Theorem 2.2. At first Friedman prepares the following auxiliary lemma. Define

$$
K(x, t ; \xi, \tau)=\frac{1}{2 \sqrt{\pi(t-\tau)}} \cdot \exp \left\{-\frac{(x-\xi)^{2}}{4(t-\tau)}\right\}
$$

Lemma 2.3. Suppose that (i) $\rho(t)(0 \leqq t \leqq \sigma)$ be a continuous function; (ii) $s(t)(0 \leqq t \leqq \sigma)$ satisfy a Lipshitz condition. Then for every $0<t \leqq \sigma$,

$$
\begin{aligned}
& \lim _{x \rightarrow s(t)-0} \frac{\partial}{\partial x} \int_{0}^{t} \rho(\tau) K(x, t ; s(\tau), \tau) d \tau \\
& \quad=\frac{1}{2} \rho(t)+\int_{0}^{t} \rho(t)\left[\frac{\partial}{\partial x} K(x, t ; s(\tau), \tau)\right]_{x=s(t)} d \tau .
\end{aligned}
$$

We introduce Green's function for the half line $x>0$ which satisfies the boundary condition $u(0, t)=0(t>0)$ :

$$
G(x, t ; \xi, \tau)=K(x, t ; \xi, \tau)-K(-x, t ; \xi, \tau) .
$$

Integrating Green's identity:

$$
\frac{\partial}{\partial \xi}\left(G \frac{\partial u}{\partial \xi}-u \frac{\partial G}{\partial \xi}\right)-\frac{\partial}{\partial \tau}(G u)=0
$$


over the domain $0<\xi<s(t), 0<\varepsilon<\tau<t-\varepsilon$ and letting $\varepsilon \rightarrow 0$, we get, on using (2.2), (2.3), (2.4),

$$
\begin{aligned}
u(x, t)= & \int_{0}^{t} u_{\xi}(s(\tau), \tau) G(x, t ; s(\tau), \tau) d \tau \\
& +\int_{0}^{t} f(\tau) G_{\xi}(x, t ; 0, \tau) d \tau+\int_{0}^{b} \psi(\xi) G(x, t ; \xi, 0) d \xi
\end{aligned}
$$

Denoting $v(\tau)=u_{\xi}(s(\tau), \tau)$, we differentiate both side of (2.7) with respect to $x$ and let $x \rightarrow s(t)-0$. Using Lemma 2.3, we obtain, on introducing the Neumann function $N(x, t ; \xi, \tau)=K(x, t ; \xi, \tau)+K(-x, t ; \xi, \tau)$ for the half line $x>0$,

$$
\begin{aligned}
v(t)= & 2[\psi(0)-f(0)] N(s(t), t ; 0,0)+2 \int_{0}^{b} \dot{\psi}(\xi) N(s(t), t ; \xi, 0) d \xi \\
& -2 \int_{0}^{t} \dot{f}(\tau) N(s(t), t ; 0, \tau) d \tau+2 \int_{0}^{t} v(\tau) G_{x}(s(t), t ; s(\tau), \tau) d \tau
\end{aligned}
$$

where, by (2.5)

$$
s(t)=b-\int_{0}^{t} v(\tau) d \tau
$$

Hencefore this integral system (2.7)-(2.9) will be denoted by FBP. I'.

Furthermore it is proved that for every solution $u, s$ of FBP. I, $v(t)$ must satisfy the integral equation (2.8), where $s(t)$ is defined by (2.9). Suppose conversely that for some $\sigma>0, v(t)(0 \leqq t<\sigma)$ is a continuous solution of the integral equation (2.8) where $s(t)$ is defined by (2.9) and $s(t)>0$. Then it is also proved that $u(x, t), s(t)$ (where $u(x, t)$ is defined by (2.7) with $u_{\xi}(s(\tau), \tau)$ replaced by $\left.v(\tau)\right)$ form a solution of FBP. I.

Regarding the integral system (2.8)-(2.9) as a nonlinear integral equation of Volterra type $v=T_{b} v\left(T_{b}:\right.$ nonlinear) and introducing an appropriate function space, the existence and uniqueness of a global solution can be proved in a standard manner as follows:

(i) A solution local in time is constructed by using that $T_{b}$ is a contraction locally with respect to the time $t$;

(ii) The local solution is continued over to a larger interval with the aid of some appropriate a priori estimates.

2.2. Here we state our main results. We denote the free boundary problem 
with $b=0$ in FBP. I by FBP. II, in which the initial condition (2.3) is removed and $(2.5 \mathrm{~b})$ is replaced by

$$
s(0)=0 \text {. }
$$

We assume that $f(t)$ satisfies the following:

Condition (f.1): $f(t)$ is continuously differentiable in $0 \leqq t<+\infty$.

Condition (f.2): $f(t)$ is non-negative for $0 \leqq t<+\infty$, and if $f(0)=0 f(t)$ satisfies the additional inequality $\dot{f}(0)>0$.

Definition 2.4. We say that $u, s$ form a solution of FBP. II for $0<t<\sigma(0<\sigma<+\infty)$ if $u, s$ satisfy conditions (i) and (ii) in Definition 2.1 and the following (iii)', (iv) ${ }^{\prime}$ and (v)' in place of (iii), (iv) and (v):

(iii) $^{\prime} \quad 0 \leqq \lim$ inf $u(x, t) \leqq \lim \sup u(x, t)<+\infty$ as $t \rightarrow 0, x \rightarrow 0$.

(iv) $)^{\prime} s(t)$ is continuous for $0 \leqq t<\sigma$ and furthermore is continuously differentiable for $0<t<\sigma$, i.e., $v(t)$ is continuous for $0<t<\sigma$. Moreover, $v$ satisfies $\int_{0+}(-v(\tau)) d \tau<+\infty$.

$(\mathrm{v})^{\prime} \quad u$ and $s$ satisfy FBP. II.

The condition (iv)' allows some singularity of $v(t)$ at $t=0$ which actually happens if $f(0)>0$. We state our results in the following.

Theorem 2.5. In FBP. II, suppose that $f(t)$ satisfies conditions $(f .1,2)$. Then (i) there exists a solution $u, s$ of FBP. II for $0<t<+\infty$. Furthermore, $s(t)$ defining the free boundary is monotone nondecreasing in t. (ii) $x=s(t)$ satisfies $c_{0} \leqq \frac{s(t)}{\sqrt{\int_{0}^{t} f(\tau) d \tau}} \leqq c_{1}$ in a neighbourhood of $t=0$, where $c_{i}(i=1,2)$ is a positive constant dependent on $f(t)$ only.

Theorem 2.5 will be proved in $\S 4$ after necessary preparations given in $\S 3$. However, we here give a brief outline of the proof of Theorem 2.5 as follows: (i) We reduce FBP. II to a nonlinear integral equation of Volterra type, (ii) For $b>0$, let $u^{b}(x, t), s^{b}(t)$ and $v^{b}(t)$ be a unique solution of FBP. I' with $\psi=\psi^{b}(x)=\frac{f(0)}{b}(b-x)(0<x<b)$. Next we make $b=b_{n} \rightarrow 0$ through an appropriate sequence $b=b_{n}(n=1,2, \ldots)$ and prove that $u^{b} \rightarrow u, s^{b} \rightarrow s$ and $v^{b} \rightarrow v$ where $u, s$ and $v$ is a solution of FBP. II'.

It should be noted that $s^{b}$ defined in (ii) satisfies the monotonicity with respect to $b$, which plays an important part in the proof of Theorem 2.5. 


\section{§3. Some Preliminaries}

As a preparation for the proof of Theorem 2.5 we state the following lemmas.

\section{1.}

Lemma 3.1. In Lemma 2.3, instead of (i) and (ii) suppose that (i)' $\rho(t)$ is continuous for $0<t \leqq \sigma$ and satisfies $\int_{0+}|\rho(\tau)| d \tau<+\infty$; (ii) $s(t)$ is continuous for $0 \leqq t \leqq \sigma$ and, moreover, continuously differentiable for $0<t \leqq \sigma$. Then (2.6) holds as it is.

Proof: We see easily that even if we use (i)', (ii)', in place of (i), (ii) in the proof [3] of Lemma 2.3, the proof goes on without modification.

Letting $b \rightarrow 0$ in FBP. $\mathrm{I}^{\prime}$ formally, we obtain the following integral system:

$$
\begin{aligned}
& u(x, t)=\int_{0}^{t} v(\tau) G(x, t ; s(\tau), \tau) d \tau+\int_{0}^{t} f(\tau) G_{\xi}(x, t ; 0, \tau) d \tau \\
& (0<x<s(t), 0<t \leqq \sigma) \\
& v(t)=-2 f(0) N(s(t), t ; 0,0)-2 \int_{0}^{t} \dot{f}(\tau) N(s(t), t ; 0, \tau) d \tau \\
& +2 \int_{0}^{t} v(\tau) G_{x}(s(t), t ; s(\tau), \tau) d \tau \quad(0<t \leqq \sigma) \\
& s(t)=-\int_{0}^{t} v(\tau) d \tau \quad(0 \leqq t \leqq \sigma),
\end{aligned}
$$

Hereafter this integral system will be denoted by FBP. II'.

Lemma 3.2. Suppose that FBP. II has a solution in a sense of Definition 2.4 and that FBP. II' has a continuous solution $u, v, s$ in each domain where they are defined. Then FBP. II is equivalent to FBP. II'.

Proof: By using the Green's identity and Lemma 3.1, it is easily shown that FBP. II is reduced to FBP. $\mathrm{II}^{\prime}$. Conversely it is proved by the same way as in Friedman's paper [3] that a solution of FBP. II' 
satisfies FBP. II in the sense of Definition 2.4.

3.2. In order to deduce a comparison theorem for FBP. I, let us consider FBP. I with $b, f$ and $\psi$ replaced by $b_{i}(>0), f_{i}$ and $\psi_{i}(x)=\frac{f_{i}(0)}{b_{i}}\left(b_{i}-x\right)$ $\left(0<x<b_{i}\right)$ for $i=1,2$, respectively. The corresponding solution will be denoted by $u_{i}(x, t)$ and $s_{i}(t)$. The functions $f_{i}$ and $\psi_{i}$ are subject to the same assumptions as $f$ and $\psi$, respectively, and hence, these solutions do exist. We have

Lemma 3.3. In addition to the assumptions as above, suppose that $f_{1}(t) \geqq f_{2}(t)(0 \leqq t \leqq \sigma)$ and $b_{1} \geqq b_{2} \geqq 0$. Then we have

$$
s_{1}(t) \geqq s_{2}(t) \quad(0 \leqq t \leqq \sigma) .
$$

Proof: The proof will be given in two steps. The first step deals with the case of $b_{1}>b_{2}$. That is,

Ist step: We claim that if $f_{1}(t) \geqq f_{2}(t)(0 \leqq t \leqq \sigma)$ and $b_{1}>b_{2}$, then we have $s_{1}(t)>s_{2}(t)(0 \leqq t \leqq \sigma)$. We use reduction to absurdity. Suppose that $s_{1}(t)$ and $s_{2}(t)$ cross each other for the first time at $t=t_{0}>0$, i.e., $s_{1}\left(t_{0}\right)$ $=s_{2}\left(t_{0}\right), s_{1}(t)>s_{2}(t)\left(0 \leqq t \leqq t_{0}\right)$. Then we have

$$
\dot{s}_{2}\left(t_{0}\right) \geqq \dot{s}_{1}\left(t_{0}\right)
$$

On the other hand, we shall compare $u_{1}(x, t)$ with $u_{2}(x, t)$ in the domain $0<x<s_{2}(t), 0<t<t_{0}$. With $w=u_{1}-u_{2}$, we obtain from FBP. I for $u_{1}$ and $u_{2}$;

$$
\begin{cases}w_{t}=w_{x x}, \quad 0<x<s_{2}(t), & 0<t<t_{0}, \\ w(0, t)=f_{1}(t)-f_{2}(t) \geqq 0, & 0<t<t_{0}, \\ w(x, 0)=\psi_{1}(x)-\psi_{2}(x)>0, & 0<x<b_{2}, \\ w\left(s_{2}(t), t\right)=u_{1}\left(s_{2}(t), t\right)>0, & 0<t<t_{0} .\end{cases}
$$

By using the strong maximum principle $[16]$ for (3.5), we get

$$
-u_{1, x}\left(s_{1}\left(t_{0}\right), t_{0}\right)>-u_{2, x}\left(s_{2}\left(t_{0}\right), t_{0}\right) .
$$

Using (2.5a) and (3.6), we get $\dot{s}_{1}\left(t_{0}\right)>\dot{s}_{2}\left(t_{0}\right)$ which contradicts (3.4). 
2nd step: We consider the general case. In fact, $s(t)$ depends continuously on $b$ in FBP. I (cf. §4). Thus, if we take $\varepsilon>0$ and denote by $x=s_{1}^{\varepsilon}(t)$ the free boundary $x=s(t)$ of FBP. I with $b$ replaced by $b_{1}^{\varepsilon}=b_{1}+\varepsilon$, we get

$$
s_{1}^{\varepsilon}(t)>s_{2}(t) \quad(0 \leqq t \leqq \sigma),
$$

by virtue of 1 st step. If we make $\varepsilon \rightarrow 0$ in (3.7), we get $s_{1}(t) \geqq s_{2}(t)$ $(0 \leqq t \leqq \sigma)$.

Lemma 3.4. In FBP. I, suppose that $f(t)$ satisfies condition (f.1) and, moreover, that either

$$
\dot{f}(t) \geqq 0(0 \leqq t \leqq \sigma) \quad \text { if } \quad f(0)>0, \quad \text { or } \quad \dot{f}(0)>0 \quad \text { if } \quad f(0)=0 .
$$

Furthermore, if we assume in particular

$$
\psi(x)=\frac{f(0)}{b}(b-x) \quad(0<x<b),
$$

then we have for the solution $u(x, t)$ and $s(t)$,

$$
-u_{x}(0, t) \geqq \frac{f(t)}{s(t)} \geqq-u_{x}(s(t), t) \quad(0 \leqq t \leqq \sigma)
$$

Proof: Putting $W(x, t)=\frac{f(t)}{s(t)}(s(t)-x) \quad(0<x<s(t), 0<t<\sigma)$, we shall compare $u(x, t)$ with $W(x, t)$ in the domain $0<x<s(t), 0<t<\sigma$. For $w=W-u$ we obtain

$$
\begin{cases}w_{t}=w_{x x}+W_{t}, & 0<x<s(t), 0<t<\sigma, \\ w(0, t)=0 & 0<t<\sigma, \\ w(x, 0)=0 & 0<x<b, \\ w(s(t), t)=0 & 0<t<\sigma,\end{cases}
$$

where $W_{t}=\dot{f}(t)\left(1-\frac{x}{s(t)}\right)+\frac{\dot{s}(t)}{s^{2}(t)} \cdot f(t) \cdot x$.

Using (3.8) and the monotonicity of $s(t)$, we see that $W_{t} \geqq 0$. Therefore the positivity of a solution of the heat equations leads to $w=W-u \geqq 0$ 
$(0<x<s(t), 0<t<\sigma)$ which implies that there hold $w_{x}(0, t) \geqq 0(0 \leqq t \leqq \sigma)$ and $w_{x}(s(t), t) \leqq 0(0 \leqq t \leqq \sigma)$. Thus we get (3.12).

Lemma 3.5. In FBP. I. let $f, \psi$ be as in Lemma 3.4. Then we have for the solution $u(x, t)$ and $s(t)$,

$$
\begin{aligned}
& K_{0} \sqrt{\int_{0}^{t} f(\tau) d \tau} \leqq s(t) \leqq b+\frac{2}{K_{0}} \sqrt{\int_{0}^{t} f(\tau) d \tau} \quad(0 \leqq t \leqq \sigma), \\
& 0<\dot{s}(t)=-v(t) \leqq \frac{1}{K_{0}} \cdot \frac{f(t)}{\sqrt{\int_{0}^{t} f(\tau) d \tau}}=\frac{2}{K_{0}} \cdot \frac{d}{d t} \sqrt{\int_{0}^{t} f(\tau) d \tau} \\
& (0<t \leqq \sigma)
\end{aligned}
$$

where $K_{0}=\frac{1}{\sqrt{1+f(\sigma)}}$.

Proof: Integrating the equation $u_{\tau}=u_{\xi \xi}$ in the domain $0<\xi<s(t)$, $0<\tau<t$ and using the initial and boundary conditions which $u$ satisfies, we obtain

$$
s(t)+\int_{0}^{s(t)} u(\xi, t) d \xi=-\int_{0}^{t} u_{\xi}(0, \tau) d \tau+b\left(1+\frac{1}{2} f(0)\right) .
$$

By the maximum principle and (3.8), we get

$$
\int_{0}^{s(t)} f(t) d \xi \geqq \int_{0}^{s(t)} u(\xi, t) d \xi \text {. }
$$

Using the first inequality of (3.10) and the monotonicity of $s(t)$, we get

$$
-\int_{0}^{t} u_{\xi}(0, \tau) d \tau \geqq \int_{0}^{t} \frac{f(\tau)}{s(\tau)} d \tau \geqq \frac{1}{s(t)} \cdot \int_{0}^{t} f(\tau) d \tau
$$

Substituting (3.15) and (3.16) into (3.14), we get

$$
(1+f(\sigma)) \cdot s(t) \geqq \frac{1}{s(t)} \int_{0}^{t} f(\tau) d \tau
$$

which implies the first inequality of (3.12);

$$
s(t) \geqq K_{0} \sqrt{\int_{0}^{t} f(\tau) d \tau} \quad(0 \leqq t \leqq \sigma) .
$$


Using (3.17) and the second inequality of (3.10) we have (3.13). Integration of both sides of (3.13) with respect to $t$ from 0 to $t$ leads to the second inequality of (3.12).

Lemma 3.6. In FBP. I, suppose that $f(0)>0$ and $\psi(x)$ satisfies (3.9). In paticular, if we take $\sigma(>0)$ sufficiently small so that there holds for $0 \leqq t \leqq \sigma$,

$$
f(t)>0
$$

then we have for the solution $u(x, t)$ and $s(t)$,

$$
\begin{array}{ll}
K_{1} \sqrt{t} \leqq s(t) \leqq b+\frac{2}{K_{1}} \cdot K_{3} \cdot \sqrt{t} & (0 \leqq t \leqq \sigma) \\
0<\dot{s}(t)=-v(t) \leqq \frac{K_{3}}{K_{1}} \cdot \frac{1}{\sqrt{t}} & (0 \leqq t \leqq \sigma)
\end{array}
$$

where $K_{1}=\min _{0 \leqq t \leqq \sigma} f(t), K_{2}=\sqrt{\frac{K_{1}}{1+K_{1}}}$ and $K_{3}=\max _{0 \leqq t \leqq \sigma} f(t)$.

Proof: Let $f(t)$ and $\psi(x)$ in FBP. I be (i) $f(t)$ which satisfies (3.18) and $\frac{f(0)}{b}(b-x)(0<x<b)$; (ii) $K_{1}$ which is the minimum of $f(t)(0 \leqq t \leqq \sigma)$ and $\frac{K_{1}}{b}(b-x)(0<x<b)$. Then we denote the free boundary $s(t)$ corresponding to (i) and (ii) by $s(t)$ and $s_{m}(t)$, respectively. By Lemmas 3.3 and 3.5 ,

$$
K_{0} \sqrt{K_{1}} \cdot \sqrt{t} \leqq s_{m}(t) \leqq s(t) \quad(0<t \leqq \sigma) .
$$

Instead of Lemma 3.4, we now claim that

$$
0<\dot{s}(t)=-v(t) \leqq \frac{K_{3}}{s(t)} \quad(0<t \leqq \sigma)
$$

Indeed, putting $W(x, t)=\frac{K_{3}}{s(t)}(s(t)-x)(0<x<s(t), 0<t<\sigma)$ and repeating the same arguments as in the proof of Lemma 3.4, we get (3.22). Using (3.21) and (3.22), we get (3.20). From (3.20) the second inequality of (3.19) follows. 


\section{§4. The Proof of Theorem}

\subsection{Construction of solution}

It is enough to prove the existence of a solution for $0 \leqq t \leqq \sigma$, for some $\sigma>0$, since we can then apply Theorem 2.2 and thus conclude the existence of a solution for $0 \leqq t<+\infty$. Furthermore, the proof of the monotonicity of $s(t)$ is similar to that given in [3] and will therefore be omitted here. Let the notation $u^{b}(x, t), s^{b}(t)$ and $v^{b}(t)$ be the same as at the end of $\S \S 2.2$.

1st step: Let $\left\{b_{n}\right\}$ be a sequence which satisfies $b_{n}>b_{n+1}>0(n=1,2, \ldots)$ and $b_{n} \rightarrow 0$ as $n \rightarrow \infty$. Then Lemma 3.3 leads to that $s^{b_{n}}(t)>s^{b_{n+1}}(t)$ $(0 \leqq t \leqq \sigma)$. As $b=b_{n} \rightarrow 0$, we see that $s^{b}(t)$ converges to $s(t)$ at every point in $0 \leqq t \leqq \sigma$. The limit function $s(t)$ satisfies $s(0)=0$.

2nd step: Consider now the family $v^{b}$ for $b=b_{n}$. Then there holds that (i) if we use Lebesgue's theorem, we get that for any $\psi \in C^{1}[0, \sigma]$, $\int_{0}^{\sigma} v^{b}(\tau) \psi(\tau) d \tau=-\int_{0}^{\sigma} s^{b}(\tau) \psi(\tau) d \tau=-s^{b}(\sigma)+b+\int_{0}^{\sigma} s^{b}(\tau) \dot{\psi}(\tau) d \tau \rightarrow-s(\sigma)$ $+\int_{0}^{\sigma} s(\tau) \dot{\psi}(\tau) d \tau \quad\left(b=b_{n} \rightarrow 0\right)$; (ii) if we take $\delta(0<\delta<\sigma)$ sufficiently small and use (3.13) and (3.20), then we see that $v^{b}$ is uniformly bounded in $L^{\infty}(\delta, \sigma)$ and, hence, is weakly* compact in $L^{\infty}(\delta, \sigma)$, while any limit function must be the derivative of $s$ (in distribution sense). Consequently, $v^{b}$ converges weakly* in $L^{\infty}(\delta, \sigma)$ to a function $v^{\infty}(t) \in L^{\infty}(\delta, \sigma)$ for every $0<\delta(<\sigma)$. Obviously, $v^{\infty}(t)$ is well defined almost everywhere in $(0, \sigma]$. Also $v^{\infty}$ is non-positive.

Then we have

$$
\lim _{b=b_{n} \rightarrow 0} \int_{0}^{\sigma} v^{b_{n}}(t) \psi(t) d t=\int_{0}^{\sigma} v^{\infty}(t) \psi(t) d t
$$

for bounded function $\psi$ whose support is in $(0, \sigma]$.

3rd step: The limit function $s(t)$ defined in 1st step is continuous in $0 \leqq t \leqq \sigma$. In fact, in view of Ascoli-Arzela's theorem and Lemmas 3.5 and 3.6 , we see that $s^{b}(t)$ converges to $s(t)$ locally uniformly in $0<t \leqq \sigma$ and $s(t)$ is continuous in $0<t \leqq \sigma$. Furthermore if we prove that $s(t) \rightarrow 0$ $(t \rightarrow+0)$, we see that $s(t)$ is continuous in $0 \leqq t \leqq \sigma$ since $s(t)$ satisfies $s(0)=0$. If we apply (3.14) to $s^{b}(t)$ and make $b=b_{n} \rightarrow 0$, then we get 


$$
K_{0} \sqrt{\int_{0}^{t} f(\tau) d \tau} \leqq s(t) \leqq \frac{2}{K_{0}} \sqrt{\int_{0}^{t} f(\tau) d \tau} \quad(0<\iota \leqq \sigma)
$$

From this $s(+0)=0$ is obvious. If $f(t)$ satisfies the assumptions of Lemma 3.6 , we may repeat the same arguments mentioned above.

Using Dini's theorem, we conclude that $s^{b}(t)$ converges uniformly to $s(t)$ in $0 \leqq t \leqq \sigma$. On the other hand, it follows from Lemmas 3.5 and 3.6 and the monotonicity of $s^{b}(t)$ that $s(t)$ is a positive and nondecreasing function in $0<t \leqq \sigma$. Finally, $s(t)$ satisfies a Lipshitz condition in $0<t \leqq \sigma$. 4th step: In 2nd step, we proved that $v^{b}$ converges weakly* to $v^{\infty} \in L_{\mathrm{loc}}^{\infty}(0$, $\sigma)$. Here we shall show that $v^{b}$ converges at every point in $0<t \leqq \sigma$ to a function which we denote by $v^{0}(t)$. If we replace $\psi(x)$ in FBP. I' by $\psi^{b}(x)=\frac{f(0)}{b}(b-x)(b>0)$, we get

$$
\begin{aligned}
v^{b}(t)= & -\frac{f(0)}{b} \int_{0}^{b} N\left(s^{b}(t), t ; \xi, 0\right) d \xi-2 \int_{0}^{t} \dot{f}(\tau) N\left(s^{b}(t), t ; 0, \tau\right) d \tau \\
& +2 \int_{0}^{t} v^{b}(\tau) G_{x}\left(s^{b}(t), t ; s^{b}(\tau), \tau\right) d \tau \quad(0 \leqq t \leqq \sigma)
\end{aligned}
$$

Making $b=b_{n} \rightarrow 0$ in (4.2), we can show

$$
\begin{aligned}
v^{b} \rightarrow v^{0}(t)= & -2 f(0) N(s(t), t ; 0,0)-2 \int_{0}^{t} \dot{f}(\tau) N(s(t), t ; 0, \tau) d \tau \\
& +2 \int_{0}^{t} v^{\infty}(\tau) G_{x}(s(t), t ; s(\tau), \tau) d \tau \quad(0<t \leqq \sigma) .
\end{aligned}
$$

Some detailed proof of (4.3) will be given in Appendix.

5th setp: Here we shall prove that $s(t) \in C[0, \sigma]$ and $v^{\infty}(t) \in L_{\mathrm{loc}}^{\infty}(0, \sigma)$ is a solution of $\mathrm{FBP}$. $\mathrm{II}^{\prime \prime}$. In 4 th step, we proved that $v^{b}(t)$ converges in any interval $0<\mu \leqq t \leqq \sigma$ to $v^{0}(t)$. From the pointwise convergence of $v^{b}(t)$ to $v^{0}(t)$ and the manner of convergence of $v^{b}$ to $v^{\infty}$, it is clear that $v^{\infty}(t)=v^{0}(t)$ almost everywhere. In other words, we have that, for almost all $t, 0<t<\sigma$,

$$
\begin{aligned}
v^{\infty}(t)= & -2 f(0) N(s(t), t ; 0,0)-2 \int_{0}^{t} f(\tau) N(s(t), t ; 0, \tau) d \tau \\
& +2 \int_{0}^{t} v^{\infty}(\tau) G_{x}(s(t), t ; s(\tau), \tau) d \tau .
\end{aligned}
$$


We now redefine $v^{\infty}(t)$ on a null set so that (4.4) holds for every $t$, i.e., $v^{\infty}(t) \equiv v^{0}(t)$. Then $v^{\infty}(t)$ is a solution of (4.4), which is continuous in $0<t \leqq \sigma$ and satisfies $\int_{0+}\left(-v^{\infty}(\tau)\right) d \tau<+\infty$.

Finally, letting $b=b_{n} \rightarrow 0$ in (2.9) and noting the uniform integrability of $v^{b}$ (see (3.13)) we find that $s(t)$ in consideration satisfies

$$
s(t)=-\int_{0}^{t} v^{\infty}(\tau) d \tau \quad(0 \leqq t \leqq \sigma)
$$

and is absolutely continuous in $0 \leqq t \leqq \sigma$. If we substitute $v=v^{\infty}(t), s(t)$ constructed above into the right side of (3.1), we see that $u(x, t)$ of $(3.1)$ is a solution of FBP. II'.

4.2. It remains to consider the behaviour of $s(t)$ as $t$ tends to zero. If we apply Lemmas 3.5 and 3.6 to $s=s^{b}(t)$ and make $b=b_{n} \rightarrow 0$, the statement of (ii) of Theorem 2.5 follows immediately.

\section{Appendix}

Here we shall prove (4.3) as follows.

(i) It follows that for $\mu \leqq t \leqq \sigma \quad(0<\mu<\sigma)$,

(A.1) $\quad \lim _{b=b_{n} \rightarrow 0}(-) \frac{2 f(0)}{b} \int_{0}^{b} N\left(s^{b}(t), t ; \xi, 0\right) d \xi=-2 f(0) N(s(t), t ; 0,0)$.

In fact, making $b=b_{n} \rightarrow 0$, the second term of the right side of the following inequality;

$$
\begin{aligned}
& \left|\frac{1}{b} \int_{0}^{b} N\left(s^{b}(t), t ; \xi, 0\right) d \xi-N(s(t), t ; 0,0) d \xi\right| \\
& \leqq \\
& \quad \frac{1}{b} \int_{0}^{b}\left|N\left(s^{b}(t), t ; \xi, 0\right)-N(\dot{s}(t), t ; \xi, 0)\right| d \xi \\
& \quad+\frac{1}{b} \int_{0}^{b}|N(s(t), t ; \xi, 0)-N(s(t), t ; 0,0)| d \xi
\end{aligned}
$$

converges to zero. Using the mean value theorem and the inequality $\sqrt{2} x e^{-x^{2}} \leqq e^{-\frac{1}{2} x^{2}}$ and making $b=b_{n}$ sufficiently small, we get that for any $\varepsilon(>0),\left|N\left(s^{b}(t), t ; \xi, 0\right)-N(s(t), t ; \xi, 0)\right|<\varepsilon$ in the domain $0<\mu \leqq t \leqq \sigma$, 
$0 \leqq \xi \leqq b_{1}$, which implies that also the first term of the right side of (A.2) converges to zero.

(ii) It follows that for $0<\mu \leqq t \leqq \sigma$,

$$
\begin{array}{r}
\lim _{b=b_{n} \rightarrow 0} \int_{0}^{t} v^{b}(\tau) G_{x}\left(s^{b}(t), t ; s^{b}(\tau), \tau\right) d \tau \\
\quad=\int_{0}^{t} v^{\infty}(\tau) G_{x}(s(t), t ; s(\tau), \tau) d \tau .
\end{array}
$$

For every fixed $t$ and $\varepsilon$, where $0<\varepsilon<t<\sigma$, we divide the third term on the right side of (4.2) into two parts, namely

$$
\int_{0}^{t}=\int_{0}^{t-\varepsilon}+\int_{t-\varepsilon}^{t}=N_{1}+N_{2}
$$

If we can prove that

$$
\lim _{b=b_{n} \rightarrow 0} N_{1}=\int_{0}^{t-\varepsilon} v^{\infty}(\tau) G_{x}(s(t), t ; s(\tau), \tau) d \tau
$$

and that uniformly with respect to $b=b_{n}$ and $t(0<\mu \leqq t \leqq \sigma)$

$$
\lim _{\varepsilon \rightarrow 0} N_{2}=0
$$

and

$$
\lim _{\varepsilon \rightarrow 0} \int_{t-\varepsilon}^{t} v^{\infty}(\tau) G_{x}(s(t), t ; s(\tau), \tau) d \tau=0
$$

then it completes the proof of (A.3). Replacing $\psi$ in (4.1) by $G_{x}(s(t)$, $t ; s(\tau), \tau) \in C[0, t-\varepsilon]$, we get

$$
\begin{array}{r}
\lim _{b=b_{n} \rightarrow 0} \int_{0}^{t-\varepsilon} v^{b}(\tau) G_{x}(s(t), t ; s(\tau), \tau) d \tau \\
=\int_{0}^{t-\varepsilon} v^{\infty}(\tau) G_{x}(s(t), t ; s(\tau), \tau) d \tau
\end{array}
$$

Using that $G_{x}\left(s^{b}(t), t ; s^{b}(\tau), \tau\right)$ converges uniformly in $0 \leqq \tau \leqq t-\varepsilon$ to $G_{x}(s(t), t ; s(\tau), \tau)$ as $b=b_{n} \rightarrow 0$ and that $\int_{0}^{t-\varepsilon}\left(-v^{b}(\tau)\right) d \tau$ is uniformly bounded by virtue of (3.13), (3.20), we get 
(A.8) $\lim _{b=b_{n} \rightarrow 0} \int_{0}^{t-\varepsilon} v^{b}(\tau)\left[G_{x}\left(s^{b}(t), t ; s^{b}(\tau), \tau\right)-G_{x}(s(t), t ; s(\tau), \tau)\right] d \tau=0$.

From (A.7) and (A.8) follows (A.4). Next, using the Lipschitz continuity of $s^{b}(t)(0 \leqq t \leqq \sigma)$ and the following inequality:

$$
\int_{t-\varepsilon}^{t} \frac{s^{b}(t)+s^{b}(\tau)}{t-\tau} \cdot K\left(-s^{b}(t), t ; s^{b}(\tau), \tau\right) d \tau \leqq \frac{1}{\sqrt{\pi}} \int_{\gamma}^{+\infty} \frac{e^{-y}}{\sqrt{y}} d y
$$

where $\gamma=\frac{s^{b}(t)^{2}}{4 \varepsilon}$, we get

$$
\int_{t-\varepsilon}^{t} v^{b}(\tau) G_{x}\left(s^{b}(t), t ; s^{b}(\tau), \tau\right) d \tau \leqq M^{2} \sqrt{\varepsilon}+A_{0} \cdot M \cdot \int_{\gamma}^{+\infty} \frac{e^{-y}}{\sqrt{y}} d y
$$

where $M=\frac{1}{K_{0}} \max _{\frac{\mu}{2} \leqq \tau \leqq \sigma} \frac{f(\tau)}{\sqrt{\int_{0}^{\tau} f(\lambda) d \lambda}} \quad\left(0<\varepsilon<\frac{\mu}{2}\right)$

and $A_{0}$ is an absolute constant.

Using (3.12), (3.19), we conclude that the right side of (A.9) tends to zero as $\varepsilon \rightarrow 0$, uniformly with respect to $t$ and $b=b_{n}$. Thus we see (A.5). On the other hand, replacing $\psi$ in (4.1) by $\left[\operatorname{sgn} v^{\infty} G_{x}\right] G_{x}(s(t), t$; $s(\tau), \tau) \in C[t-\varepsilon, t-\delta]$ for any $0<\delta<\varepsilon$, we get

$$
\begin{aligned}
& \int_{t-\varepsilon}^{t-\delta}\left|v^{\infty}(\tau) G_{x}(s(t), t ; s(\tau), \tau)\right| d \tau \\
& \leqq \lim _{b=b_{n} \rightarrow 0} \sup \int_{t-\varepsilon}^{t-\delta}\left|v^{b}(\tau) G_{x}(s(t), t ; s(\tau), \tau)\right| d \tau \\
& \leqq M \int_{t-\varepsilon}^{t-\delta}\left|G_{x}(s(t), t ; s(\tau), \tau)\right| d \tau \\
& \leqq M^{2} \sqrt{\varepsilon}+A_{0} \cdot M \cdot \int_{\beta}^{+\infty} \frac{e^{-y}}{\sqrt{y}} d y
\end{aligned}
$$

where $\beta=\frac{s(t)^{2}}{4 \varepsilon}$. The last inequality follows as (A.9) with $s^{b}$ replaced by s. In fact, $v^{b}$ is uniformly bounded in $L^{\infty}\left(\frac{\mu}{2}, \sigma\right)\left(0<\delta<\varepsilon<\frac{\mu}{2}\right)$ by (3.13) and (3.20), i.e.,

$$
0<-v^{b} \leqq M \quad\left(\frac{\mu}{2} \leqq t \leqq \sigma\right)
$$


which implies

$$
\left|s^{b}(t)-s^{b}(\tau)\right| \leqq M \cdot|t-\tau| \quad\left(\frac{\mu}{2} \leqq \tau \leqq t \leqq \sigma\right) .
$$

From (A.12), we get

$$
|s(t)-s(\tau)| \leqq M \cdot|t-\tau| \quad\left(\frac{\mu}{2} \leqq \tau \leqq t \leqq \sigma\right),
$$

since $s^{b}(t)$ converges uniformly to $s(t)$ in $0 \leqq t \leqq \sigma$. Since the right side of (A.10) does not depend on $\delta$ and tends to zero as $\varepsilon \rightarrow 0$, the integral on the left side of (A.10) is convergent for $\delta=0$ and is bounded by the right side of (A.10). We now use the results of 3rd step and conclude that if $0<\mu<t<\sigma$ then the right side of (A.10) tends to zero as $\varepsilon \rightarrow 0$, uniformly with respect to $t$ and $b=b_{n}$. Hence follows (A.6).

(iii) The second integral on the right side of (4.2) can be dealt with quite similarly.

\section{Acknowledgements}

The author wishes to express his gratitude to Prof. H. Fujita for frequent, stimulating, and helpful discussions.

\section{References}

[1] Rubinstein, L.I., On the determination of the portion of the boundary which separates two phases in the one dimensional problem of Stefan, Doklady Akad. Nauk SSSR (N.S.), 58 (1947), 217-220.

[2] Dacev, A.B., On the linear problem of Stefan. The case of two phases of infinite thickness, Doklady Akad. Nauk SSSR (N.S.), 74 (1950), 445-448.

[3] Friedman, A., Free boundary problems for parabolic equations I. Melting of solids, J. Math. Mech., 8, (1959), 499-518.

[4] Evans II, G.W., A note on the existence of a solution to a problem of Stefan, Quart. Appl. Math., 9 (1951), 185-193.

[5] Douglas J. and Gallie, T.M., On the numerical integration of a parabolic differential equation subject to a moving boundary condition, Duke Math. J., 22 (1955), 557-571.

[6] Sestini, G., Esistenza di una soluzione in problemi analoghi a quello di Stefan, Rivista Mat. Univ. Parma, 3 (1952), 3-23.

[7] Miranker, W.I., A free boundary value problem for the heat equation, Quart. Appl. Math., 6 (1958), 121-130. 
[8] Douglas, J., A uniqueness theorem for the solution of a Stefan problem, Proc. Amer. Math. Soc., 8 (1957), 402-408.

[9] Kyner, W.T., An existence and uniqueness theorem for a nonlinear Stefan problem, J. Math. Mech., 9 (1959), 483-498.

[10] Friedman, A., Remarks on Stefan-type free boundary problems for parabolic equations, J. Math. Mech., 9 (1960), 885-903.

[11] Kolodner, I.I., Free boundary problem for the heat equation with applications to problems of change of phase, Comm. Pure Appl. Math., 9 (1956), 1-31.

[12] Ladyzenskaja, O., Solonnikov, V., and Uralceva, N., Linear and quasilinear equations of parabolic type, "Nauka", Moscow, 1967: English transl, Math. Monographs, Vol. 23, Amer. Math. Soc., Providence, R.I., 1968.

[13] Oleinik, O., On the Stefan-type free boundary problems for parabolic equations, Seminari 1962-1963 Anal. Alg. Geom. e Topol., 1, 388-403. Ist. Naz. Acta. Mat., Ed. Cremonese.

[14] Brezis, H., On Some degenerate nonlinear parabolic equations, Proc. sympos. Appl. Math., 18, (1968), 28-38 Amer. Math. Soc., Providence, R.I.

[15] Nogi, T., Difference methods for the Stefan-type Problem, to appear.

[16] Friedman, A., Partial differential equations of parabolic type, Prentice-Hall, Englewood Cliffs, N. J., 1964. 
